\title{
HS-1793, a resveratrol analogue, induces cell cycle arrest and apoptotic cell death in human breast cancer cells
}

\author{
JIN-AH KIM ${ }^{1}$, DONG HWAN KIM ${ }^{1}$, MOHAMMAD AKBAR HOSSAIN ${ }^{1,2}$, \\ MIN YOUNG KIM ${ }^{1}$, BOKYUNG SUNG ${ }^{1}$, JEONG-HYUN YOON ${ }^{1}$, HONGSUK SUH $^{3}$, \\ TAE CHEON JEONG ${ }^{4}$, HAE YOUNG CHUNG ${ }^{1}$ and NAM DEUK KIM ${ }^{1}$ \\ ${ }^{1}$ Department of Pharmacy, Molecular Inflammation Research Center for Aging Intervention, \\ Pusan National University, Busan 609-735, Republic of Korea; ${ }^{2}$ Department of Pharmacology \\ and Toxicology, Faculty of Pharmacy, Umm Al-Qura University, Makkah, Kingdom of Saudi Arabia; \\ ${ }^{3}$ Department of Chemistry and Chemistry Institute for Functional Materials, Pusan National University, \\ Busan 609-735; ${ }^{4}$ College of Pharmacy, Yeungnam University, Gyeongsan 712-749, Republic of Korea
}

Received August 29, 2013; Accepted October 30, 2013

DOI: 10.3892/ijo.2013.2207

\begin{abstract}
Resveratrol, a polyphenolic compound, is a naturally occurring phytochemical and is found in a variety of plants, including food such as grapes, berries and peanuts. It has gained much attention for its potential anticancer activity against various types of human cancer. However, the usefulness of resveratrol as a chemotherapeutic agent is limited by its photosensitivity and metabolic instability. In this study the effects of a synthetic analogue of resveratrol, HS-1793, on the proliferation and apoptotic cell death were investigated using MCF-7 (wild-type p53) and MDA-MB-231 (mutant p53) human breast cancer cells. HS-1793 inhibited cell growth and induced apoptotic cell death in a concentration-dependent manner. The induction of apoptosis was determined by morphological changes, cleavage of poly(ADP-ribose) polymerase, alteration of $\mathrm{Bax} / \mathrm{Bcl}-2$ expression ratio and caspase activities. Flow cytometric analysis revealed that HS-1793 induced $\mathrm{G} 2 / \mathrm{M}$ arrest in the cell cycle progression in both types of cells. Of note, HS-1793 induced p53/p21 ${ }^{\text {WAFI/CIPI }}$-dependent apoptosis in MCF-7 cells, whereas it exhibited p53-independent apoptosis in MDA-MB-231 cells. Furthermore, HS-1793 showed more potent anticancer effects in several aspects compared to resveratrol in MCF-7 and MDA-MB-231 cells. Thus, these findings suggest that HS-1793 has potential as a candidate chemotherapeutic agent against human breast cancer.
\end{abstract}

Correspondence to: Professor Nam Deuk Kim, Department of Pharmacy, College of Pharmacy, Pusan National University, 63 Beongil 2, Busandaehag-ro, Geumjeong-gu, Busan 609-735, Republic of Korea

E-mail: nadkim@pusan.ac.kr

Key words: resveratrol analogue, breast cancer cells, apoptosis, cell cycle

\section{Introduction}

Breast cancer is one of the most common malignant tumors and is the leading cause of cancer-related deaths in women worldwide (1). Five-year survival rate for tumor confined to the breast has increased to $\sim 80-90 \%$ over the last decade. However, approximately one-third of breast cancer patients still die from the disease once tumor metastasized to other organs (2).

Major treatment strategies for breast cancer consist, either separately or in combination of, radiotherapy, surgery and chemotherapy (3). Many agents used to treat breast cancer are often associated with severe systemic toxicities. Acquired tumor drug resistance also limits their use in the treatment of breast cancer. Therefore, novel non-toxic therapeutic agents active against breast cancer are under investigation, with the need to develop new agents acting on novel targets.

Resveratrol (trans-3,4,5'-trihydroxydtilbene, Fig. 1A) is a natural polyphenol compound $(4,5)$. It has been reported to exhibit a wide range of biological and pharmacological properties. It exists in two isoforms: trans-resveratrol and cis-resveratrol; however, the trans-isomer is more stable than cis-resveratrol. Resveratrol-glucuronide is the major form absorbed when compared to the very minute amounts of unconjugated resveratrol and resveratrol sulfate are absorbed (6). Resveratrol has been reported to induce apoptosis in various cancerous or transformed cells in culture, chemically induced mouse skin tumors, and in transplanted tumors in nude mice by activating both extrinsic and intrinsic pathways of cell death machinery $(7,8)$. Resveratrol has shown to inhibit three major stages of carcinogenesis: initiation, promotion and progression (9). However, exposure to high doses of resveratrol is required to induce apoptosis in cancer cells. In addition, resveratrol is photosensitive and metabolically unstable, with stilbene double bonds being readily oxidized $(3,10)$.

In previous studies, the resveratrol analog HS-1793 (Fig. 1B) overcame the resistance conferred by Bcl-2 in U937 leukemia cells (11). In addition, HS-1793 induced higher anti-tumor activity in various cancer cell lines (12-14) and inhibited hypoxia- 
induced HIF-1 and VEGF expressions (15). However, detailed apoptosis mechanisms at work are not yet well understood. Therefore, the purpose of the present study was to investigate the anti-proliferation and anti-apoptotic potential of HS-1793 and to evaluate the signal pathway involved in relation to wild-type or mutant p53 protein in human breast cancer cells, such as MCF-7 (wild-type p53) and MDA-MB-231 (mutant p53) cells.

\section{Materials and methods}

Chemicals. trans-3,4,5'-Trihydroxystilbene (resveratrol) was purchased from Sigma-Aldrich Co. (St. Louis, MO, USA). 4-(6-Hydroxy-2-naphthyl)-1,3-benzendiol (HS-1793) was supplied by Professor Hongsuk Suh (Pusan National University, Busan, Korea), and dissolved at a concentration of $100 \mathrm{mM}$ in ethanol as a stock solution, and stored $-20^{\circ} \mathrm{C}$. The stock solution was diluted with cell culture medium to the desired concentration prior to use. The maximal concentration of ethanol did not exceed $0.1 \%(\mathrm{v} / \mathrm{v})$ in the treatment range, where there was no influence on the cell growth.

Cell culture.MCF-7 (wild-type p53) and MDA-MB-231 (mutant type p53) cells were obtained from American Type Culture Collection (Manassas, VA, USA). MCF-7 and MDA-MB-231 cells were maintained in DMEM medium (HyClone, Logan, UT, USA) in humidified atmosphere of $37^{\circ} \mathrm{C}$ with $5 \% \mathrm{CO}_{2}$. DMEM supplemented with $10 \%$ heat-inactivated fetal bovine serum (FBS, HyClone), $2 \mathrm{mM}$ glutamine (Sigma-Aldrich), $100 \mathrm{U} / \mathrm{ml}$ penicillin (HyClone) and $100 \mu \mathrm{g} / \mathrm{ml}$ streptomycin (HyClone).

MTT assay and growth inhibition. Cell survival was determined by colorimetric 3-(4,5-dimethylthiazol-2-yl)2,5-diphenyltetrazolium bromide (MTT) assay which measures mitochondrial activity in viable cells. Cells $\left(1.5 \times 10^{5}\right)$ were plated in each well of a 6 -well plate, allowed to adhere overnight and then the culture medium was replaced with fresh media. Cells were treated with resveratrol or HS-1793 at concentrations of $12.5,25$ and $50 \mu \mathrm{M}$ for $24 \mathrm{~h}$. Control groups were treated with ethanol equal to the highest percentage of $(<0.1 \%)$ solvent used in experimental conditions for MTT assay. After $24 \mathrm{~h}$ the medium was replaced with fresh medium. MTT was freshly prepared at $5 \mathrm{mg} / \mathrm{ml}$ in PBS and passed through a filter (pore size, $0.2 \mu \mathrm{m}$ ). An aliquot of $2 \mathrm{ml}$ of MTT stock solution was added to each well, and the plate was incubated at $37^{\circ} \mathrm{C}$ for $4 \mathrm{~h}$ in humidified $5 \% \mathrm{CO}_{2}$ incubator. After $4 \mathrm{~h}$, media were removed and formazan crystals were dissolved in $2 \mathrm{ml}$ of DMSO for 10 min with gentle agitation. The optical density of each well was measured with a spectrophotometer equipped with a 540-nm filter.

Protein preparation and western blot analysis. Cells were harvested and solubilized in lysis buffer $(40 \mathrm{mM}$ Tris, $\mathrm{pH} 8.0$, $120 \mathrm{mM} \mathrm{NaCl}, 0.5 \% \mathrm{NP}-40,0.1 \mathrm{mM}$ sodium orthovanadate, $2 \mu \mathrm{g} / \mathrm{ml}$ aprotinin, $2 \mu \mathrm{g} / \mathrm{ml}$ leupeptin and $100 \mu \mathrm{g} / \mathrm{ml}$ phenylmethylsulfonyl fluoride), and the supernatant was collected and protein concentrations were then measured with a Bio-Rad protein assay kit (Bio-Rad, Hercules, CA, USA) with bovine serum albumin as the standard. Equal amount of protein extracts were denatured by boiling at $100^{\circ} \mathrm{C}$ for

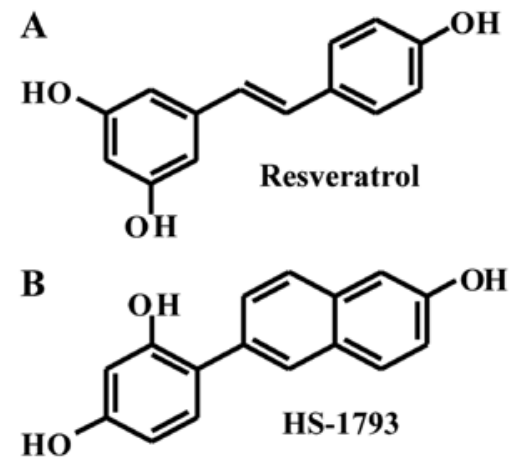

Figure 1. Chemical structures of resveratrol and HS-1793.

5 min in sample buffer $(0.5 \mathrm{M}$ Tris-Cl, pH 6.8, $4 \%$ SDS, $20 \%$ glycerol, $0.1 \%$ bromophenol blue, $10 \% \beta$-mercaptoethanol) in ratio of 1:1. Equal amount of the total proteins were subjected to 6-15\% SDS-polyacrylamide gel electrophoresis (PAGE) and transferred to polyvinylidene difluoride (PVDF) membrane. The membranes were blocked with $5 \%$ non-fat dry milk in TBS-T buffer $(20 \mathrm{mM}$ Tris, $100 \mathrm{mM} \mathrm{NaCl}, \mathrm{pH} 7.5$ and $0.1 \%$ Tween-20) for $1 \mathrm{~h}$ at room temperature. The membrane was incubated with diluted primary antibody in TBS-T buffer at $4^{\circ} \mathrm{C}$ overnight. The membranes were washed once for 10 min 3 times with TBS-T buffer and incubated for $1 \mathrm{~h}$ with secondary antibody in TBS-T buffer at room temperature.

Hoechst staining and observation of nuclear structure. MCF-7 and MDA-MB-231 cells were incubated with 12.5, 25 and $50 \mu \mathrm{M}$ resveratrol or HS-1793 for $24 \mathrm{~h}$ and then cells were washed twice with PBS containing $1 \%$ bovine serum albumin (PBS-B) and fixed with 70\% ethanol containing $0.5 \%$ Tween -20 at $4^{\circ} \mathrm{C}$ for $30 \mathrm{~min}$. Fixed cells were washed with PBS-B, and stained with $4 \mu \mathrm{g} / \mathrm{ml}$ Hoechst 33342 for $30 \mathrm{~min}$ at room temperature. The stained cells were washed twice with PBS-B and observed under Zeiss Axiophot microscope (Göttingen, Germany) at x400 magnification.

Flow cytometric analysis. Cells were trypsinized, washed with PBS, and fixed in 95\% ethanol with Tween-20 at $4^{\circ} \mathrm{C}$ overnight. Prior to analyses, cells were again washed with PBS, suspended in cold propidium iodide (PI, Sigma-Aldrich) solution, and incubated at room temperature for $30 \mathrm{~min}$ in the dark. Before analysis cell suspensions were filtered with $40 \mu \mathrm{M}$ pore nylon mesh to remove debris. Flow cytometry analysis was performed on a FACScan (Becton Dickinson, San Jose, CA, USA).

Immunoprecipitation and western blot analysis. Total cell lysates were lysed in lysis buffer. The supernatant was collected and protein concentration determined with Bio-Rad protein assay kit (Bio-Rad). For immunoprecipitation, cell extracts were incubated with immunoprecipitating antibody in lysis buffer at $4^{\circ} \mathrm{C}$ for $1 \mathrm{~h}$. The immuno-complexes were precipitated with protein A-sepharose beads (Sigma-Aldrich) for $1 \mathrm{~h}$, and washed five times with extraction buffer prior to boiling in SDS sample buffer. Immunoprecipitated proteins or aliquots containing $40 \mu \mathrm{g}$ protein were separated on SDS-PAGE and transferred to PVDF membranes. Western 
Table I. Effect of resveratrol or HS-1793 on cell cycle phase distribution of MCF-7 and MDA-MB-231 cells. ${ }^{\mathrm{a}}$

\begin{tabular}{|c|c|c|c|c|c|c|c|c|c|}
\hline \multirow[b]{2}{*}{ Cells } & \multirow[b]{2}{*}{ Phase } & \multicolumn{4}{|c|}{ Resveratrol } & \multicolumn{4}{|c|}{ HS-1793 } \\
\hline & & Control & $12.5 \mu \mathrm{M}$ & $25 \mu \mathrm{M}$ & $50 \mu \mathrm{M}$ & Control & $3.0 \mu \mathrm{M}$ & $6.25 \mu \mathrm{M}$ & $12.5 \mu \mathrm{M}$ \\
\hline \multirow[t]{3}{*}{ MCF-7 } & G0/G1 (\%) & 52.6 & 28.4 & 63.1 & 70.5 & 52.6 & 84.0 & 62.2 & 13.8 \\
\hline & $\mathrm{S}(\%)$ & 41.7 & 71.6 & 35.5 & 29.5 & 41.7 & 10.0 & 20.1 & 18.8 \\
\hline & G2/M (\%) & 5.7 & 0 & 1.4 & 0 & 5.7 & 6.0 & 17.7 & 67.4 \\
\hline \multirow[t]{3}{*}{ MDA-MB-231 } & G0/G1 (\%) & 54.0 & 36.6 & 55.0 & 69.1 & 54.0 & 50.4 & 39.0 & 9.3 \\
\hline & $\mathrm{S}(\%)$ & 40.8 & 62.6 & 45.0 & 30.9 & 40.8 & 36.5 & 30.8 & 26.5 \\
\hline & G2/M (\%) & 5.2 & 0.8 & 0 & 0 & 5.2 & 13.1 & 30.2 & 64.2 \\
\hline
\end{tabular}

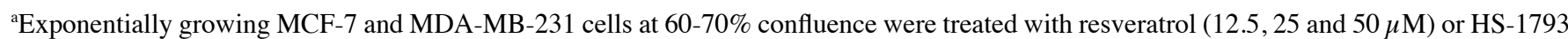
(3, 6.25 and $12.5 \mu \mathrm{M})$ for $24 \mathrm{~h}$ and cell cycle distribution was analyzed using flow cytometer.

A

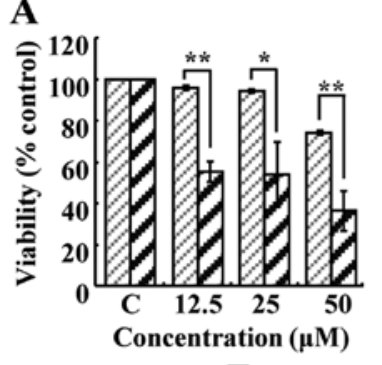

曰 Resveratrol
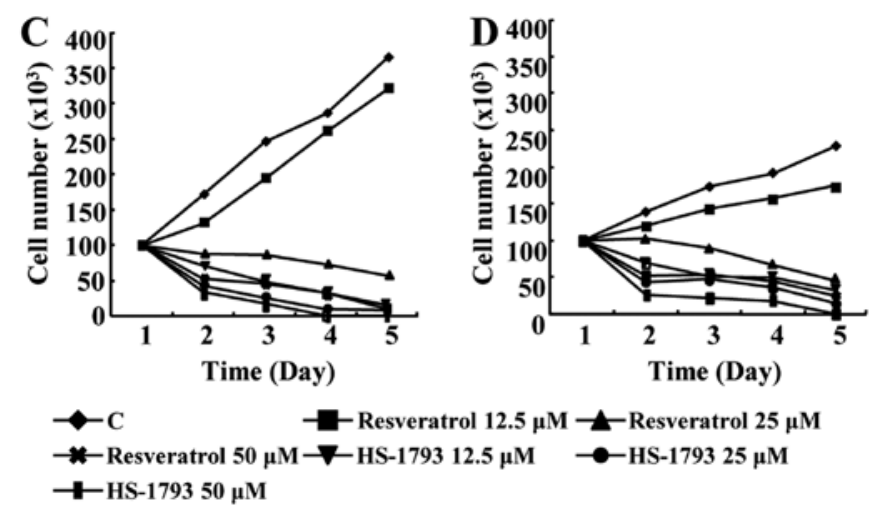

Figure 2. Effects of HS-1793 on cell viability and proliferation of MCF-7 and MDA-MB-231 cells. MCF-7 (A) and MDA-MB-231 (B) cells were treated with different concentrations of resveratrol or HS-1793 for $24 \mathrm{~h}$ and the cell survival was determined using the MTT assay. C, control. MCF-7 (C) and MDA-MB-231 (D) cells were treated with different concentrations of resveratrol or HS-1793 for 5 days and cell numbers were determined by the trypan blue exclusion method using a hematocytometer. Results are expressed as percentages of the vehicle-treated control $\pm \mathrm{SD}$ of triplicate experiments The significance was determined by Student's t-test ( $\mathrm{p}<0.05$ and ${ }^{* *} \mathrm{p}<0.01$ compared between resveratrol or HS-1793 treated cells).

blot analysis was performed. Primary antibodies to $\mathrm{p} 53$, MDM2, p21 ${ }^{\text {WAFI/CIPI }}$, cyclin D1, CDK4, CDK6, cyclin B1, Cdc2, Cdc25C, Fas, Fas-L, PARP, Bax, Bcl-2, ERK1/2, pERK, JNK and pJNK were purchased from Santa Cruz Biotechnology Inc. (Santa Cruz, CA, USA). Primary antibody to $\beta$-actin was purchased from Sigma-Aldrich. The proteins were visualized with enhanced chemiluminescence (ECL) detection system (GE Healthcare Biosciences, Pittsburgh, PA, USA).
Caspase activity assay. Cells were harvested and washed twice in PBS at $4^{\circ} \mathrm{C}$. Total cells were lysed with the lysis buffer at $4^{\circ} \mathrm{C}$ for $30 \mathrm{~min}$ with vortexing. Cell lysate $(200 \mu \mathrm{g})$ was mixed with assay buffer in a final volume of $100 \mu \mathrm{l}$, followed by addition of $10 \mu \mathrm{l}$ of $2 \mathrm{mM}$ of p-nitroaniline (pNA)-conjugated caspase-8 (Z-IETD-pNA), caspase-9 (Ac-LEHD-pNA), or caspase-3 (Z-DEVD-pNA) substrates, respective, for the caspase assay. The reaction mixture was incubated at $37^{\circ} \mathrm{C}$ for $30 \mathrm{~min}$ and the liberated pNA was measured at $405 \mathrm{~nm}$.

Statistical analysis. Results are expressed as the mean \pm SD of three separate experiments and analyzed by Student's t-test. Means were considered significantly different at $p<0.05$ or $\mathrm{p}<0.01$.

\section{Results}

HS-1793 suppresses proliferation of MCF-7 and MDA-MB231 cells. To investigate the effects of resveratrol and HS-1793 on the viability of MCF-7 and MDA-MB-231 cells, the MTT assay was performed. Resveratrol did not show any prominent effects, with $\mathrm{IC}_{50}$ values not being measurable at the concentration of $12.5,25$ and $50 \mu \mathrm{M}$. However, the $\mathrm{IC}_{50}$ values of HS-1793 in MCF-7 and MDA-MB-231 cells were $25 \mu \mathrm{M}$ and $50 \mu \mathrm{M}$, respectively (Fig. 2A and B). Therefore, HS-1793 seems to induce more efficient inhibition of cell viability than resveratrol. Cell proliferation was also evaluated by counting dead and live cell numbers by the trypan blue exclusion method. Results indicated that resveratrol and HS-1793 exerted time- and concentration-dependent inhibition of cell proliferation in both MCF-7 and MDA-MB-231 cells (Fig. 2C and D). Although both resveratrol and HS-1793 exhibited antiproliferative effect on both breast cancer cells, HS-1793 was more potent than resveratrol.

HS-1793 modulates the cell cycle in MCF-7 and MDA-MB231 cells. We next investigated whether resveratrol and HS-1793 affect cell cycle progression. Cells were treated with either resveratrol $(12.5,25$ and $50 \mu \mathrm{M})$ or HS-1793 $(3,6.25$ and $12.5 \mu \mathrm{M}$ ) for $24 \mathrm{~h}$ and then the cell cycle was analyzed by flow cytometric analysis. As shown in Table I, HS-1793 inhibited the cell growth through $\mathrm{G} 2 / \mathrm{M}$ phase arrest, while resveratrol 

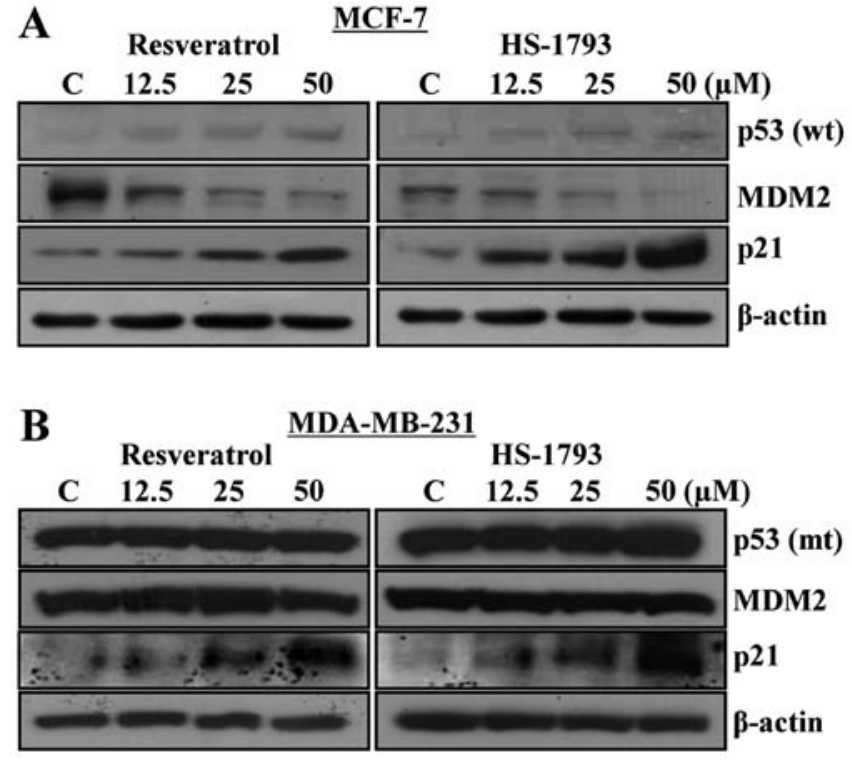

Figure 3. Effects of resveratrol and HS-1793 on the levels of cell cycle regulatory proteins such as $\mathrm{p} 53, \mathrm{MDM} 2$ and $\mathrm{p} 21^{\text {WAFI/CIPI }}$ in MCF-7 and MDAMB-231 cells. MCF-7 (A) and MDA-MB-231 (B) cells were treated with various concentration of resveratrol or HS-1793 for $24 \mathrm{~h}$. Total cell lysates were prepared and subjected to $10-12 \%$ SDS-PAGE and electrophoretically transferred to PVDF membranes. Western blot analysis was conducted with indicated antibodies and ECL kits. Representative results from three independent experiments are shown. Actin was used as a loading control. C, control.

led to G1 phase arrest in MCF-7 (16) and MDA-MB-231 (17) cells as already published.

HS-1793 modulates the cell cycle regulatory proteins in MCF-7 and MDA-MB-231 cells. Generally, p53 is known as a tumor suppressor gene and controls the expression of $\mathrm{p}^{2} 1^{\text {WAFII }}$ ${ }^{C I P I}$, a potent cyclin-dependent kinase (CDK) inhibitors in G1 and $\mathrm{G} 2 / \mathrm{M}$ phases. MDM2, an oncogene, negatively regulates p53 through inhibiting the transactivation activity of p53 by binding to its transactivation domain. MDM2 has also a ubiquitin ligase activity that leads to the degradation of p53 $(18,19)$. In MCF-7 cells, p53 and p21 ${ }^{\text {WAFI/CIPI }}$ were increased while MDM2 was decreased by both resveratrol and HS-1793. In contrast, in MDA-MB-231 cells, p21 ${ }^{\text {WAFI/CIPI }}$ was increased without a change in the level of MDM2. Given that MDA-MB231 cells are p53 mutant, there might be a p53-independent stimulus inducing the increase in $\mathrm{p} 21^{\text {WAFI/CIPI }}$ (Fig. 3A and B). Western blot analyses were conducted to further characterize the molecular mechanisms by which resveratrol or HS-1793 inhibits cell growth. The levels of intracellular proteins of G1 phase, such as cyclin D1, CDK4 and CDK6, were not significantly changed (Fig. 4A). Immunoprecipitation was conducted to investigate binding activity of the cyclin D1-CDK4 complex which showed a decrease of the complex in both cell types (Fig. 4B). Cyclin B1, Cdc2 and Cdc25C, which are proteins of G2 phase, were decreased in both cell lines by HS-1793 treatment in a concentration-dependent manner (Fig. 4C). Cdc2 and cyclin B1 interaction was also inhibited by HS-1793 treatment (Fig. 4D).

HS-1793 induces apoptotic cell death in MCF-7 and MDA-MB-231 cells. To determine whether the growth inhibi-
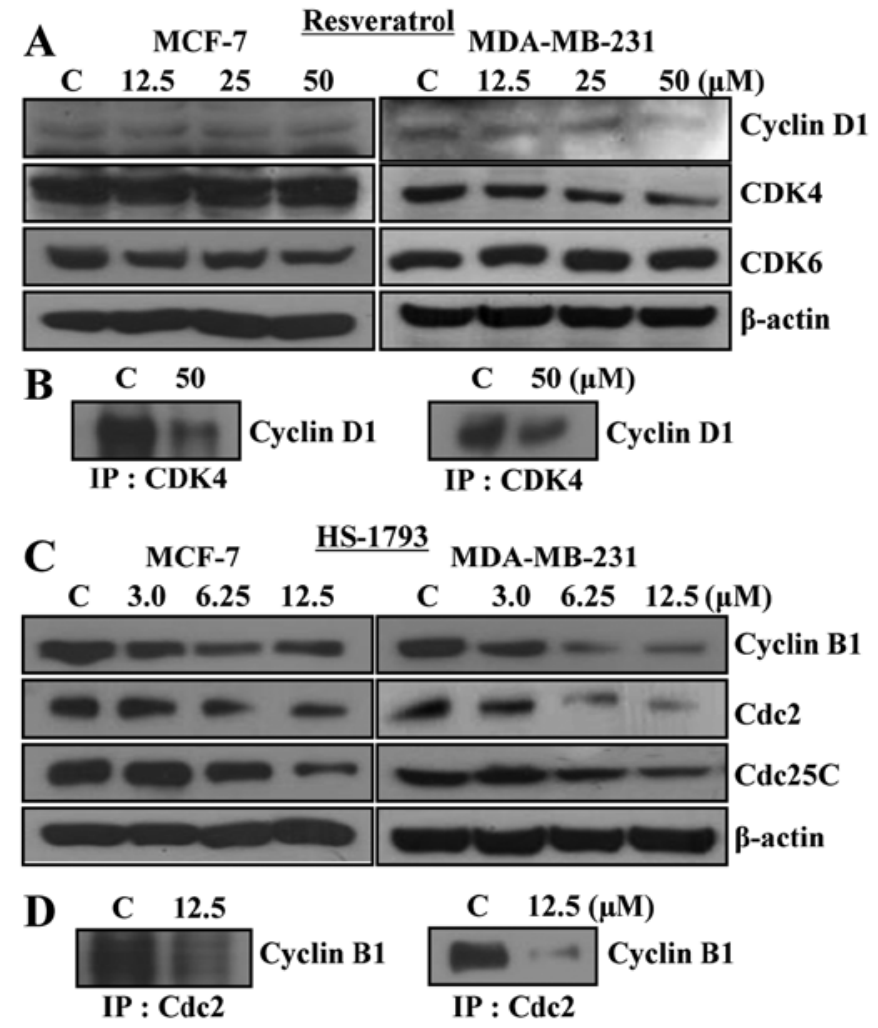

Figure 4. Effects of resveratrol and HS-1793 on the protein levels of cyclins and CDKs in MCF-7 and MDA-MB-231 cells. (A) To detect the protein levels of cell cycle regulators in G1 phase such as cyclin D1, CDK4 and CDK6, MCF-7 and MDA-MB-231 cells were treated with various concentration of resveratrol for $24 \mathrm{~h}$, collected, lysed and then cellular proteins were separated and immunoblotted. (B) After treatment with resveratrol for $24 \mathrm{~h}$, cell lysates were immunoprecipitated with anti-CDK4 antibody, separated on SDS-PAGE, transferred on to PVDF membrane, cyclin D1 protein levels were detected with anti-cyclin D1 antibody and ECL detection system. (C) To detect the protein levels of cell cycle regulators in $\mathrm{G} 2 / \mathrm{M}$ phase such as cyclin B1, Cdc2 and Cdc25C, MCF-7 and MDA-MB-231 cells were treated with various concentration of HS-1793 for $24 \mathrm{~h}$, collected, lysed and then cellular proteins were separated and immunoblotted. (D) After treatment with HS-1793 for $24 \mathrm{~h}$, cell lysates were immunoprecipitated with anti-Cdc2 antibody, separated on SDS-PAGE, transferred to PVDF membranes, cyclin B1 protein levels were detected with anti-cyclin B1 antibody and ECL detection system. Representative results from three independent experiments are shown. Actin was used as a loading control. C, control.

tory effects of resveratrol and HS-1793 could be attributed to apoptotic cell death in MCF-7 and MDA-MB-231 cells, the morphological changes were assessed with Hoechst 33342 staining (Fig. 5A and B). The control cells displayed typical normal nuclear structure in a concentrationdependent manner, while cells treated with resveratrol and HS-1793 exhibited chromosomal condensation and formation of apoptotic bodies, indicating apoptotic cell death. At $12.5 \mu \mathrm{M}, \mathrm{HS}-1793$ was effective in inducing chromosomal condensation in both cell types, whereas resveratrol did not (Fig. 5A and B). Therefore, these results also showed that HS-1793 exhibited more efficient induction of apoptosis than resveratrol in both cell lines.

HS-1793 modulates the expression levels of apoptosis-related proteins in MCF-7 and MDA-MB-231 cells. The degradation of polypeptides, such as poly(ADP-ribose) polymerase 
A

\section{MCF-7}

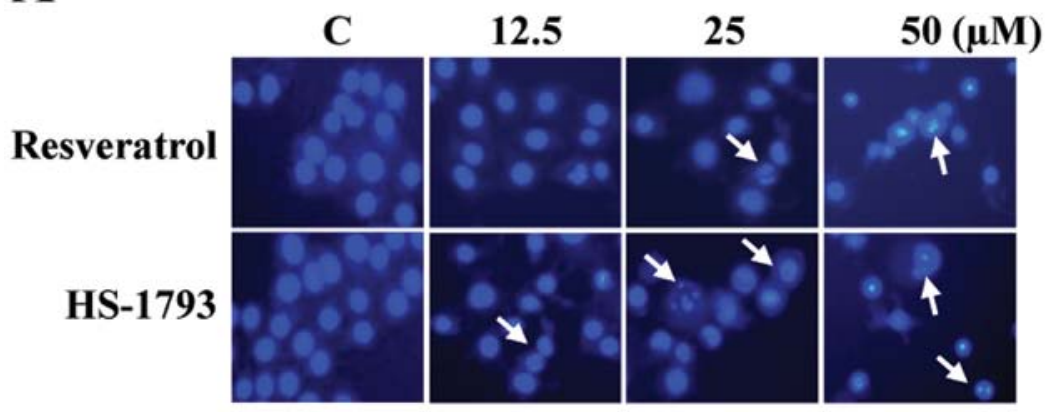

B

MDA-MB-231

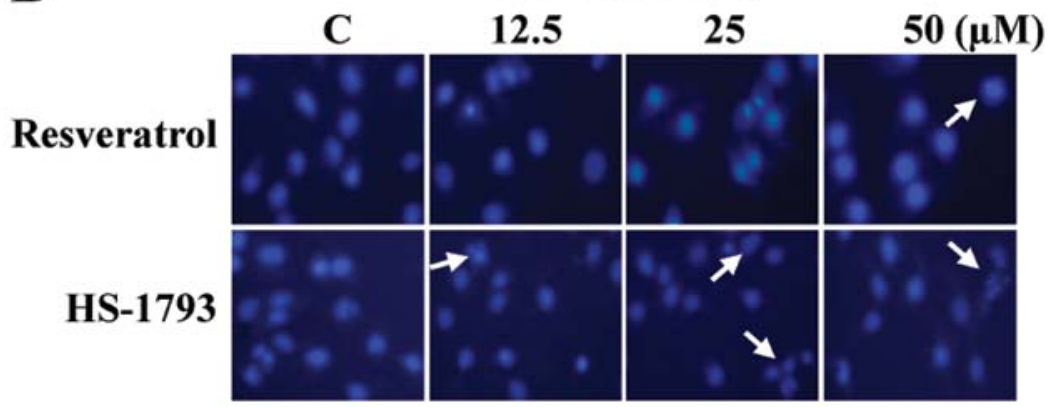

Figure 5. Morphological changes of nuclear structures in MCF-7 and MDA-MB-231 cells. MCF-7 (A) and MDA-MB-231 (B) cells were treated with resveratrol or HS-1793 at 12.5, 25 and $50 \mu \mathrm{M}$ for $24 \mathrm{~h}$. Detection of apoptotic morphology by staining with the fluorescent DNA-binding dye Hoechst 33342 . Apoptotic cells (arrows). C, control. Magnification, x400.

A

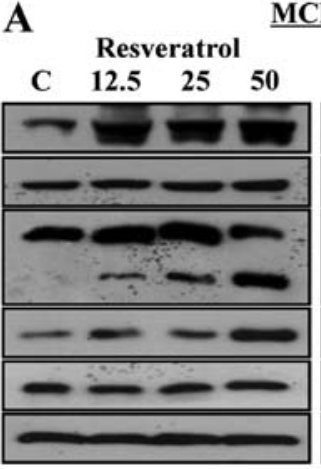

MCF-7

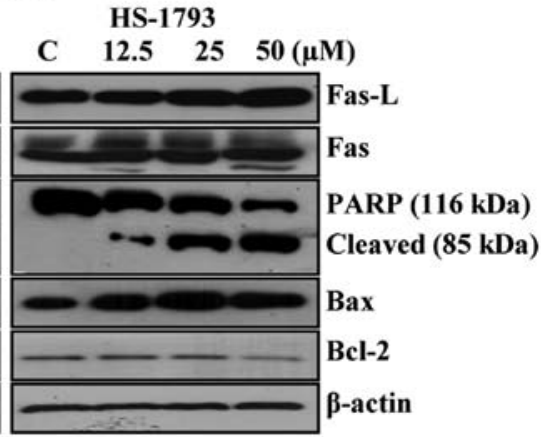

B

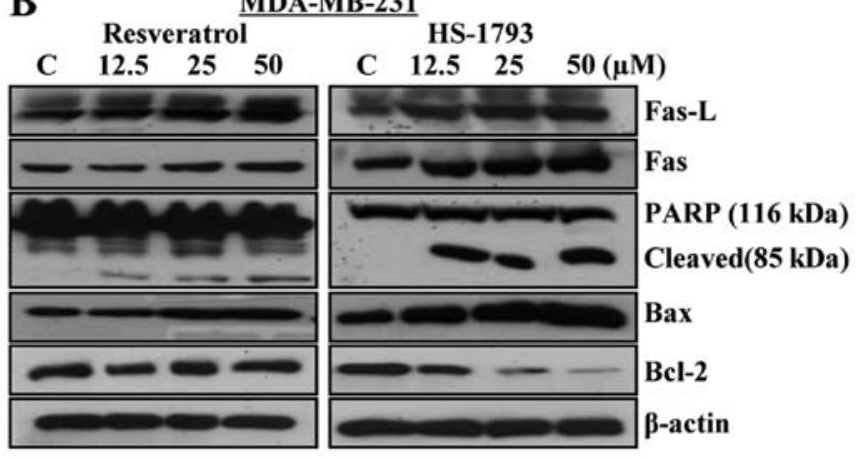

Figure 6. Effects of resveratrol and HS-1793 on the levels of apoptosis-related proteins in MCF-7 and MDA-MB-231 cells. MCF-7 (A) and MDA-MB-231 (B) cells were treated with resveratrol or HS-1793 at $12.5,25$ and $50 \mu \mathrm{M}$ for $24 \mathrm{~h}$. Total cell lysates were prepared and subjected to 6-12\% SDS-PAGE and electrophoretically transferred to PVDF membranes. Western blot analysis was conducted with the indicated antibodies and ECL kits. Representative results from three independent experiments are shown. Actin was used as a loading control. C, control.
(PARP), was examined to further study the possible involvement of apoptosis-associated caspases in the induction of apoptotic cell death (Fig. 6). Treatment with resveratrol and HS-1793 caused increase in cleavage of PARP in both cell types (Fig. 6A and B). To determine whether the expression levels of death receptors and death receptor-mediated apoptotic proteins were changed by resveratrol and HS-1793, western blot analysis was performed and expression levels of Fas, Fas-ligand (Fas-L), Bcl-2 and Bax were measured. The expression of Fas and Fas- $\mathrm{L}$ was increased in a concentration-dependent manner in both cell lines. In addition, in both HS-1793 treated cell lines, the expression level of Bcl-2 protein was markedly downregulated, while Bax was upregulated in a concentration-dependent manner. These data suggest that resveratrol and HS-1793 induce apoptosis through the alteration in expression levels of death receptor proteins as well as altered the expression ratio of $\mathrm{Bax} / \mathrm{Bcl}-2$ protein (Fig. 6A and B).

$H S-1793$ increases the caspase activity in MCF-7 and $M D A-M B-231$ cells. In an attempt to further characterize the molecular mechanisms of apoptosis induced by resveratrol or HS-1793, the activity of caspases (-3, -8, and -9) was determined by colorimetric assay. In case of MCF-7 cells (caspase- 3 null type), the activity of caspase- 8 and -9 was increased with the treatment of resveratrol or HS-1793 (Fig. 7A and B). In MDA-MB-231 cells, however, caspase-3, -8 , and -9 were all activated with the treatment of both compounds (Fig. 7C and D). Overall, these results implicate that both HS-1793 and resveratrol induce caspase-dependent apoptotic cell death in MCF-7 and MDA-MB-231 cells. 

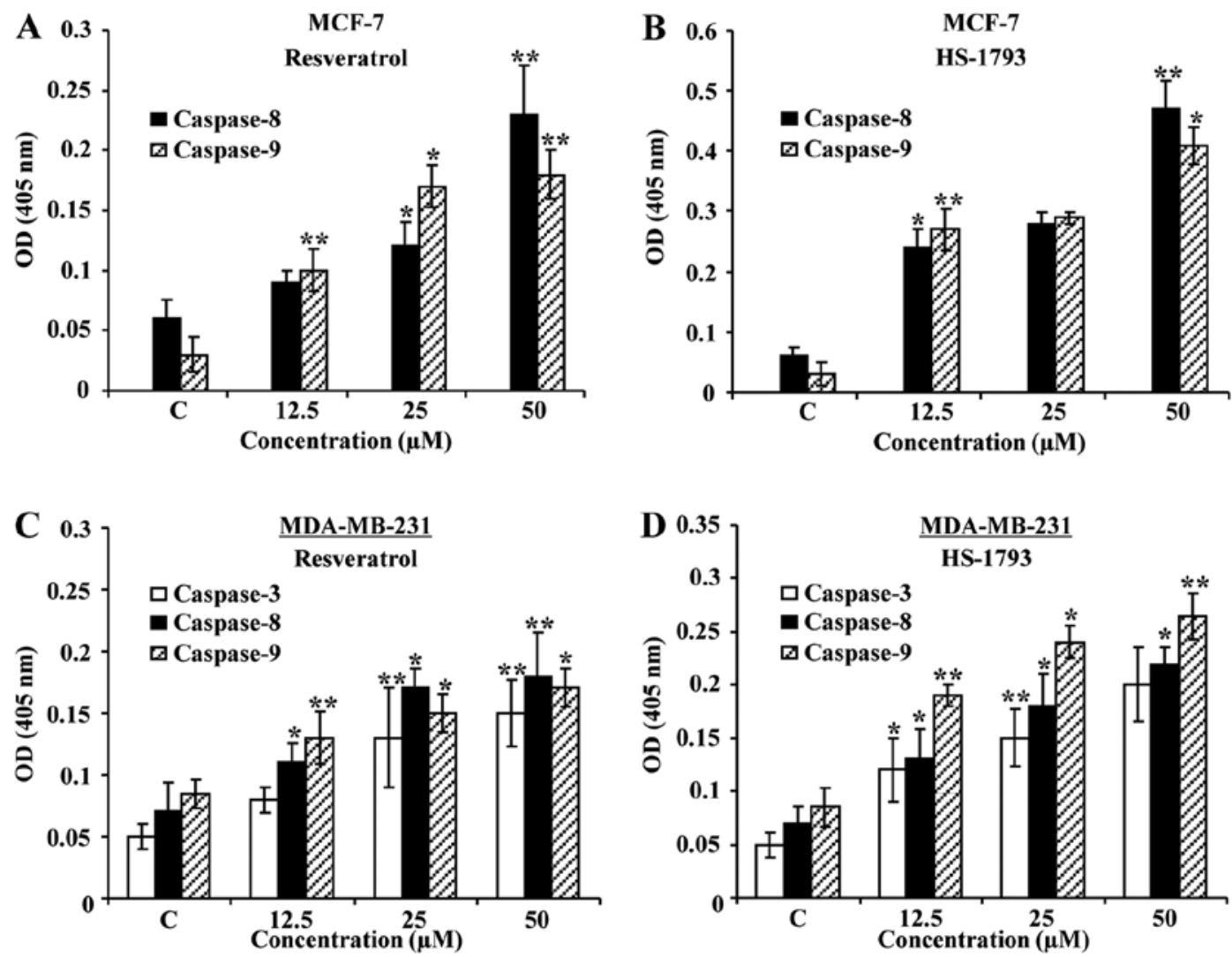

Figure 7. Effects of resveratrol and HS-1793 on the caspase activity in MCF-7 and MDA-MB-231 cells. MCF-7 (A) and MDA-MB-231 (B) cells were pretreated with various concentrations of resveratrol or HS-1793 for $24 \mathrm{~h}$. Cell lysates were prepared and then caspase-3, -8 or -9 colorimetric substrate was added (DEVD-pNA, IETD-pNA or LEHD-pNA, respectively) and the caspase activitywas measured. The results are presented as the mean \pm SD of triplicate experiments. The significance was determined by Student's t-test ( $\mathrm{p}<0.05$ and ${ }^{* *} \mathrm{p}<0.01$ compared with control cells). C, control.

A
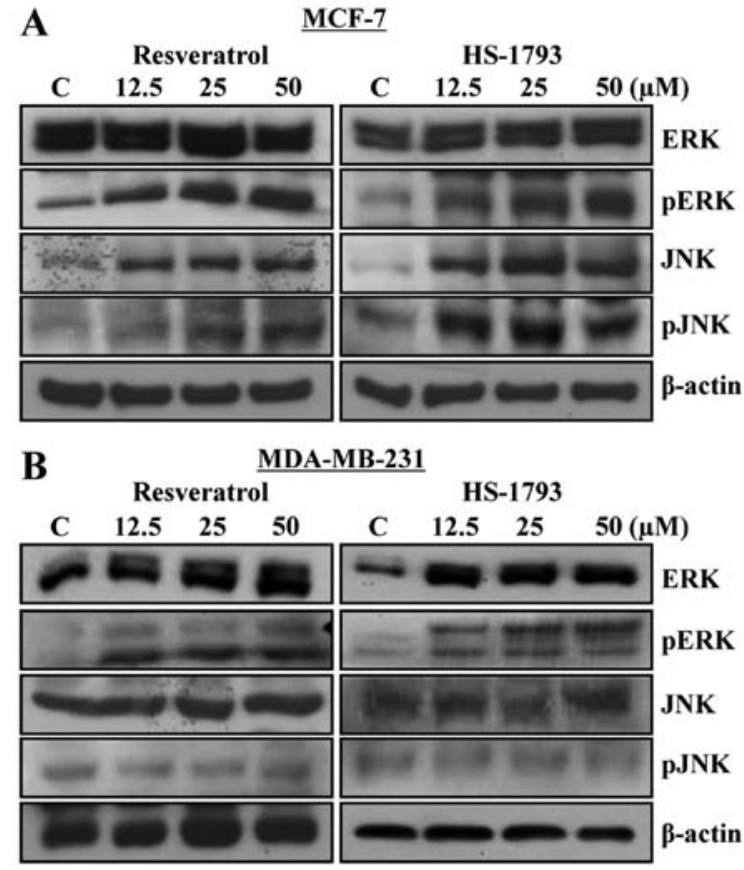

Figure 8. Effects of resveratrol and HS-1793 on the levels of MAP kinases in MCF-7 and MDA-MB-231 cells. MCF-7 (A) and MDA-MB-231 (B) cells were treated with resveratrol or HS-1793 at $12.5,25$ and $50 \mu \mathrm{M}$ for $24 \mathrm{~h}$, respectively. Total cell lysates were prepared and subjected to $10-12 \%$ SDS-PAGE and transferred to PVDF membranes. Western blot analysis was conducted with the indicated antibodies and ECL kits. Representative results from three independent experiments are shown. Actin was used as a loading control. C, control.
$H S-1793$ modulates the expression of MAP kinases in MCF-7 and $M D A-M B-231$ cells. The mitogen-activated protein kinase (MAPK) signaling pathway has been shown to play important roles in cell cycle and apoptosis $(20,21)$. Thus, to investigate whether the MAPK pathway is involved in HS-1793 and resveratrol-induced apoptosis, MCF-7 and MDA-MB-231 cells were treated with resveratrol or HS-1793 at 12.5, 25 and $50 \mu \mathrm{M}$ for $24 \mathrm{~h}$ and then the expression levels of MAPKs [i.e., extracellular signal-regulated kinase (ERK) and c-Jun N-terminal kinase (JNK)] were compared by western blot analysis. As shown in Fig. 8, both resveratrol and HS-1793 induced phosphorylation of ERK and JNK in MCF-7 cells (Fig. 8A). In contrast to this, only ERK phosphorylation was increased in MDA-MB-231 cells (Fig. 8B). These results suggest that apoptosis induced by resveratrol or HS-1793 may be mediated by different pathways in p53 wild and mutant type cell lines.

\section{Discussion}

This study was conducted to investigate and compare the effects of resveratrol and HS-1793 on the proliferation and apoptotic cell death in MCF-7 and MDA-MB-231 human breast cancer cells. The resveratrol or HS-1793 treatment in both cell lines efficiently inhibited cell growth in a concentration-dependent manner. At equimolar concentrations, HS-1793 showed more potent effects than resveratrol. Flow cytometric analysis revealed that HS-1793 induced cell cycle arrest more efficiently than resveratrol in both cell types. 
Resveratrol modulated the cell cycle progression and caused G1 phase arrest in both cell lines. However, HS-1793 treatment induced G2/M arrest and apoptosis by downregulating cyclins and CDKs with upregulations of Bax, p53 and p21 WAFI/CIPI in both cell lines.

The progression of eukaryotic cell cycle involves sequential activation of CDKs whose association with corresponding regulatory cyclins is necessary for their activations. For instance, the G1/S transition is regulated by complexes formed by cyclin D and CDK4 or CDK6 (22). The CDK inhibitors can negatively regulate cell cycle progression by competing with cyclin D1 for binding with CDK4 or CDK6 complexes and inhibiting the kinase activities of CDKs/cyclin complexes (23). In this study, the intracellular protein levels of $\mathrm{G} 1$ phase regulatory proteins such as cyclin D1, CDK4 and CDK6 were downregulated in both cell lines by resveratrol. We found that $\mathrm{G} 2$ phase regulatory protein such as cyclin B1, Cdc2 and Cdc25C were downregulated in both cell lines by HS-1793. The resveratrol analogue HS-1793 also inhibited formation of the $\mathrm{Cdc} 2 /$ cyclin B complex. Binding to cyclin B and phosphorylation at threonine 161 by CDK-activating kinase are required to activate $\mathrm{Cdc} 2$ during $\mathrm{G} 2$ and the $\mathrm{Cdc} 2 /$ cyclin B complex is kept inactive by phosphorylation on tyrosine 15 and threonine 14 of $\mathrm{Cdc} 2$ by the kinases Wee1 and Myt1, respectively (24). Although detailed mechanism of HS-1793 on Cdc2/cyclin B complex or each component was not investigated, it is likely that HS-1793, either directly or through downregulation of protein level, targets the $\mathrm{Cdc} 2 /$ cyclin B complex. The tumor suppressor protein p53, was increased in MCF-7 cells by both resveratrol and HS-1793. However, treatments with resveratrol or HS-1793 upregulated the expression level of the CDK inhibitor $\mathrm{p} 21^{\text {WAFI/CIPI }}$ in a p53-dependent and -independent manner in MCF-7 and MDA-MB-231 cells, respectively.

The resveratrol or HS-1793 treatment also induced apoptosis as demonstrated by the formation of apoptotic bodies and cleavages of PARP. Cellular p53 accumulation induces Fas-mediated apoptosis by transcriptional activation of Fas gene and by cell surface trafficking of Fas (25). In this study, induction of apoptosis by resveratrol and HS-1793 was associated with the upregulation of Fas and Fas-L in MCF-7 and MDA-MB-231 cells. The Bcl-2 family proteins play critical roles in the induction of apoptosis. Treatment with resveratrol and HS-1793 induced alterations in expression ratio of Bax protein and Bcl-2 in both cell types. During apoptosis, a series of proteolytic cleavages of various intracellular polypeptides are initiated by the action of a unique family of cysteinedependent proteases called caspases (26). We observed induction of caspase activity in both cell lines. Induction of the JNK and p38 MAPK-governed phosphorylative cascades has been reported to be involved in the mechanisms of apoptosis triggered by resveratrol (27). We found that pJNK and pERK were increased by resveratrol and HS-1793 in MCF-7 cells, whereas only pERK was increased in MDA-MB-231 cells. However, further experiments are required to clarify the detailed molecular mechanisms of action in both cell lines.

In conclusion, this study demonstrated that HS-1793 was capable of inhibiting cell proliferation and inducing apoptosis in MCF-7 and MDA-MB-231 cells harboring different p53 status. HS-1793 induced G2/M arrest and apoptosis by downregulating cyclins and CDKs with upregulation of Bax, p53, and p2 $1^{\text {WAFI/CIPI }}$ in both cell lines. The effects were mediated via either a p53-dependent or -independent pathway. Moreover, HS-1793 showed more potent effect than resveratrol on the cytotoxicity of MCF-7 and MDA-MB-231 breast cancer cells. Collectively, these results imply that HS-1793 could be a good candidate as a new potent chemotherapeutic agent against human breast cancer.

\section{Acknowledgements}

This study was supported by Basic Science Research Program through the National Research Foundation of Korea (NRF) funded by the Ministry of Education, Science and Technology (2012R1A1A2006753).

\section{References}

1. Siegel R, Naishadham D and Jemal A: Cancer statistics, 2013. CA Cancer J Clin 63: 11-30, 2013.

2. DeSantis C, Siegel R, Bandi P and Jemal A: Breast cancer statistics, 2011. CA Cancer J Clin 61: 409-418, 2011.

3. Kim HJ, Yang KM, Park YS, et al: The novel resveratrol analogue HS-1793 induces apoptosis via the mitochondrial pathway in murine breast cancer cells. Int J Oncol 41: 1628-1634, 2012.

4. Baur JA and Sinclair DA: Therapeutic potential of resveratrol: the in vivo evidence. Nat Rev Drug Discov 5: 493-506, 2006.

5. Marques FZ, Markus MA and Morris BJ: Resveratrol: cellular actions of a potent natural chemical that confers a diversity of health benefits. Int J Biochem Cell Biol 41: 2125-2128, 2009.

6. Khan N, Afaq F and Mukhtar H: Cancer chemoprevention through dietary antioxidants: progress and promise. Antioxid Redox Signal 10: 475-510, 2008.

7. Fulda S and Debatin KM: Resveratrol-mediated sensitisation to TRAIL-induced apoptosis depends on death receptor and mitochondrial signalling. Eur J Cancer 41: 786-798, 2005.

8. Garvin S, Ollinger K and Dabrosin C: Resveratrol induces apoptosis and inhibits angiogenesis in human breast cancer xenografts in vivo. Cancer Lett 231: 113-122, 2006.

9. Jang M, Cai L, Udeani GO, et al: Cancer chemopreventive activity of resveratrol, a natural product derived from grapes. Science 275: 218-220, 1997.

10. Cai YJ, Wei QY, Fang JG, et al: The 3,4-dihydroxyl groups are important for trans-resveratrol analogs to exhibit enhanced antioxidant and apoptotic activities. Anticancer Res 24: 999-1002, 2004.

11. Jeong SH, Lee JS, Jeong NY, et al: A novel resveratrol analogue HS-1793 treatment overcomes the resistance conferred by Bcl-2 and is associated with the formation of mature PML nuclear bodies in renal clear cell carcinoma Caki-1 cells. Int J Oncol 35: 1353-1360, 2009.

12. Jeong MH, Yang KM, Choi YJ, et al: Resveratrol analog, HS-1793 enhance anti-tumor immunity by reducing the $\mathrm{CD} 4{ }^{+} \mathrm{CD} 25^{+}$regulatory $\mathrm{T}$ cells in FM3A tumor bearing mice. Int Immunopharmacol 14: 328-333, 2012.

13. Jeong NY, Yoon YG, Rho JH, et al: The novel resveratrol analog HS-1793-induced polyploid LNCaP prostate cancer cells are vulnerable to downregulation of Bcl-xL. Int $\mathrm{J}$ Oncol 38: 1597-1604, 2011.

14. Jeong SH, Song IS, Kim HK, et al: An analogue of resveratrol HS-1793 exhibits anticancer activity against MCF-7 cells via inhibition of mitochondrial biogenesis gene expression. Mol Cells 34: 357-365, 2012

15. Kim DH, Hossain MA, Kim MY, et al: A novel resveratrol analogue, HS-1793, inhibits hypoxia-induced HIF-1alpha and VEGF expression, and migration in human prostate cancer cells. Int J Oncol 43: 1915-1924, 2013.

16. MaZ, Molavi O, Haddadi A,Lai R, Gossage RA and Lavasanifar A: Resveratrol analog trans 3,4,5,4'-tetramethoxystilbene (DMU-212) mediates anti-tumor effects via mechanism different from that of resveratrol. Cancer Chemother Pharmacol 63: 27-35, 2008.

17. Kotha A, Sekharam M, Cilenti L, et al: Resveratrol inhibits Src and Stat 3 signaling and induces the apoptosis of malignant cells containing activated Stat3 protein. Mol Cancer Ther 5: 621-629, 2006. 
18. Momand J, Zambetti GP, Olson DC, George D and Levine AJ: The mdm-2 oncogene product forms a complex with the p53 protein and inhibits p53-mediated transactivation. Cell 69: $1237-1245,1992$.

19. Honda R, Tanaka H and Yasuda H: Oncoprotein MDM2 is a ubiquitin ligase E3 for tumor suppressor p53. FEBS Lett 420: 25-27, 1997.

20. Makin G and Dive C: Modulating sensitivity to drug-induced apoptosis: the future for chemotherapy? Breast Cancer Res 3 : 150-153, 2001.

21. Wilkinson MG and Millar JB: Control of the eukaryotic cell cycle by MAP kinase signaling pathways. FASEB J 14: 2147-2157, 2000.

22. Bates S, Bonetta L, MacAllan D, et al: CDK6 (PLSTIRE) and CDK4 (PSK-J3) are a distinct subset of the cyclin-dependent kinases that associate with cyclin D1. Oncogene 9: 71-79, 1994.
23. Ellis M, Chew YP, Fallis L, et al: Degradation of p27(Kip) cdk inhibitor triggered by Kaposi's sarcoma virus cyclin-cdk6 complex. EMBO J 18: 644-653, 1999.

24. Taylor WR and Stark GR: Regulation of the G2/M transition by p53. Oncogene 20: 1803-1815, 2001.

25. Bennett M, Macdonald K, Chan SW, Luzio JP, Simari R and Weissberg P: Cell surface trafficking of Fas: a rapid mechanism of p53-mediated apoptosis. Science 282: 290-293, 1998.

26. Earnshaw WC, Martins LM and Kaufmann SH: Mammalian caspases: structure, activation, substrates, and functions during apoptosis. Annu Rev Biochem 68: 383-424, 1999.

27. Filomeni G, Graziani I, Rotilio G and Ciriolo MR: trans-Resveratrol induces apoptosis in human breast cancer cells MCF-7 by the activation of MAP kinases pathways. Genes Nutr 2: 295-305, 2007. 